\title{
La génétique moléculaire ne laisse même plus les ossements tranquilles
}

Dans la nuit du 16 au 17 juillet 1918, à Iekaterinburg dans la maison Ipatiev, en plein cœur de la Russie centrale, le Tsar Nicolas II, sa femme la Tsarine Alexandra, leurs quatre filles Olga, Tatiana, Maria et Anastasia ainsi que leur seul fils Alexei, furent massacrés par les Bolcheviks sur ordre de Lénine, avec trois de leurs domestiques et leur médecin de famille, le Docteur Eugeny Botkin. Pendant le massacre, quelques bijoux cachés s'échappèrent des vêtements des membres de la famille royale, exaspérant les miliciens qui défigurèrent alors les victimes à coups de crosse et de baïonnette. Les corps des victimes furent ensuite placés dans un camion pour être enterrés dans une mine. Toutefois, un incident mécanique obligea les meurtriers à creuser une fosse de fortune au bord de la route. Ils y jetèrent les corps, les recouvrirent d'acide sulfurique et refermèrent le trou à la hâte avec de la terre. Ce récit est celui rapporté en 1919 par le juge Nikolai Sokolov, un Russe blanc monarchiste, et bien qu'il ait servi de base à la version historique de la disparition des Romanov, il n'a jamais été formellement prouvé. A l'aide de matériels d'archives, deux historiens amateurs russes, Gely Ryabov et Alexander Avdonin, ont découvert à $35 \mathrm{~km}$ d'Iekaterinburg une fosse contenant des restes de squelettes humains. Les os étaient très endom- tifier neuf corps. Tous les squelettes montraient des traces de violence avant la mort, avec notamment une destruction du massif facial des crânes, ce qui a rendu les techniques d'identification faciale classiques difficiles. Certains d'entre eux avaient des dents en or, platine et porcelaine, indiquant qu'il s'agissait au moins d'aristocrates. Les experts de médecine légale russes ont conclu à l'aide des méthodes classiques d'investigation que la fosse pouvait contenir le Tsar, la Tsarine, et trois de leurs cinq enfants. En 1992, le gouvernement russe a demandé à une équipe anglo-russe de vérifier l'authenticité de l'identification des squelettes en utilisant des techniques basées sur l'étude de l'ADN. Le résultat de ce travail vient d'être publié dans le numéro de février de Nature Genetics [1].

Dans un premier temps, l'ADN a été extrait à l'aide de phénol et de chloroforme à partir de la poudre d'os, après action de protéinase $\mathrm{K}$ et de Tween, avec un rendement de l'ordre de 50 à $100 \mathrm{pg}$ d'ADN pour $1 \mathrm{~g}$ d'os. Afin de minimiser les possibilités de contamination, ces extractions ont été effectuées dans des conditions d'isolement draconiennes, sous hotte, dans un laboratoire différent de celui où étaient réalisées l'amplification et l'analyse des produits d'amplification. Dans un deuxième temps, trois approches ont été utilisées, toutes basées sur l'amplification génique in vitro
(PCR). La première a consisté à déterminer le sexe des squelettes en amplifiant le gène de l'amélogénine, une protéine de la matrice extracellulaire de l'émail des dents. Ce gène est présent à la fois sur le chromosome $\mathrm{X}$ et sur le chromosome $Y$, avec toutefois une différence de taille selon le chromosome due à une différence de taille des introns. Utilisant un couple d'amorces engendrant un fragment de 106 paires de bases spécifique du chromosome X, et de 112 paires de bases pour le chromosome Y, les chercheurs ont confirmé les conclusions des examens physiques, à savoir la présence de quatre hommes et cinq femmes. La deuxième approche a utilisé l'amplification de cinq microsatellites tétramériques afin de prouver un lien de parenté entre les corps. Ces séquences sont extrêmement polymorphes et la caractérisation des différents allèles correspondant à des tailles différentes a permis de montrer un groupe familial parmi les squelettes, constitué de deux parents et trois enfants. Tout lien de parenté avec et entre les autres corps a été exclu. Les corps non apparentés correspondraient à ceux des trois domestiques et du Docteur Botkin. La dernière approche a consisté à analyser l'ADN mitochondrial (ADNmt) pour déterminer la relation des squelettes avec les descendants par voie maternelle de la famille Romanov. En effet, l'ADNmt est de transmission 
exclusivement maternelle et, comme de multiples copies du génome mitochondrial existent par cellules, la probabilité de trouver une copie intacte dans des prélèvements anciens est accrue [2]. Trois cent quatre-vingts bases ont été étudiées dans la première région hypervariable, et 360 dans la deuxième. La comparaison des séquences obtenues à partir des neuf squelettes retrouve six séquences différant entre elles par une moyenne de six bases avec une séquence identique pour la présumée Tsarine et les trois enfants. L'ADNmt du Prince Philippe, Duc d'Edimbourg, actuellement en vie et petit-neveu par voie maternelle de la Tsarine Alexandra, s'est avéré identique à celui de la Tsarine et des trois enfants, confirmant la filiation. En revanche, les ADNmt de deux collatéraux par voie maternelle du Tsar (arrière-arrière-petit-fils et arrière-arrière-petite-fille de Louise de Hesse-Cassel, la grand-mère de Nicolas II) se sont avérés identiques entre eux, mais ils présentent une différence à la base 16169 avec celui du présumé Tsar. En effet, celui-ci présente une hétéroplasmie, avec un $\mathrm{C}$ et un $\mathrm{T}$ dans un rapport de 3,4: 1 , alors que les collatéraux ne présentent qu'un $\mathrm{T}$ à cette position. Etant donné que l'on ne connaît pas le temps nécessaire pour qu'une hétéroplasmie se transforme en homoplasmie dans la descendance de l'ADNmt non codant, ce résultat est d'interprétation délicate. Sur la base de leurs tests, les auteurs estiment à $98,5 \%$ la probabilité que les ossements trouvés soient ceux des Romanov. Toutefois, les arguments du contexte non génétique rendent cette hypothèse quasi formelle [2].

E.D.

1. Gill P, Ivanov PL, Kimpton C, Piercy R, Benson N, Tully G, Evett I, Hagelberg E, Sullivan $K$. Identification of the remains of the Romanov family by DNA analysis. Nature (ienet $1994 ; 6: 130-5$.

2. Debenham PG. Genetics leaves no bones unturned. Nature Genet 1994; 6: 113-4.

$m / s n^{\circ}$ 6-7 vol. 10, juin-juillet 94

口u BRÈVES

- Entrée sélective des protéines dans la voie de sécrétion. La majorité des protéines nouvellement synthétisées est exportée dans des vésicules qui se forment à la membrane du réticulum endoplasmique (RE). On admet généralement que toutes les protéines dépourvues de signaux de rétention quittent le RE, emportées par un flux membranaire permanent. I)ans ce modèle, la vitesse de transport d'une protéine dépend de la cinétique de son repliement, de son oligomérisation et de son association avec des molécules chaperones. La sortie de la voie d'exocytose ne s'effectue pas avant l'arrivée dans le réseau transgolgien et est limitée aux protéines possédant des signaux de ciblage identifiant leur destination finale. Une alternative à ce modèle, proposée il y a une quinzaine d'années, avait gardé la faveur de certains biologistes cellulaires et vient à nouveau occuper le devant de la scène scientifique: le transport hors du RE pourrait être limité aux protéines porteuses de signaux adéquats. Grâce à la microscopie électronique et à un système de reconstitution in vitro des premières étapes de la sortie du RE, le transport de la glycoprotéine du virus de la stomatite vésiculeuse (VSV-G) a pu être analysé quantitativement. VSV$G$ est concentrée 5 à 10 fois dans des vésicules de 40 à $80 \mathrm{~nm}$ en partance pour l'appareil de Golgi. A l'inverse, une protéine très abondante qui recircule entre le RE et les premiers saccules golgiens est moins concentrée dans ces vésicules que dans le RE. Cela constitue la preuve de l'individualisation puis de la concentration de VSV-G dans des structures vésiculaires au moment de sa sortie du RE. L'accumulation de VSV-G dans les vésicules prégolgiennes est la seule étape de concentration de cette protéine durant le transport jusqu'au réseau transgolgien. A la lumière de ces résultats, il est clair que la sortie du RE ne s'effectue pas exclusivement par le jeu du flux membranaire. Un niveau de contrôle supplémentaire assure, dans le RE, l'entrée sélective de certaines protéines dans la voie de sécrétion.

[Balch WE, et al. Cell 1994; 76: 841-52.]

Les cytochromes P450 (CYP) sont les produits d'une superfamille de gènes [1]. Deux d'entre eux sont déficients chez $5 \%$ à $10 \%$ des sujets de populations européennes, le CYP 2C19 et le CYP 2D6. Ce dernier métabolise des médicaments comme la débrisoquine, la spartéine, le dextrométhorphane, de nombreux médicaments psychotropes ou cardiovasculaires. Les sujets déficients en CYP 2D6 sont définis comme métaboliseurs lents. A l'inverse, $1 \%$ à $2 \%$ des sujets sont classés métaboliseurs ultra-rapides. L'analyse de deux familles suédoises de tels sujets a montré que le nombre de copies du gène était multiplié par 2 (famille I) et même par 12 (famille II) [2]. C'est le premier cas décrit d'effet de dosage génique ayant une conséquence phénotypique dans le métabolisme des médicaments. Il semble, en outre, que la capacité de métabolisation soit proportionnelle au nombre de gènes. Plusieurs explications ont été avancées pour expliquer cette multiplication des gènes (CYP 2I)6, mais aucune n'a été confirmée expérimentalement. Ainsi, la grande variabilité d'expression d'un gène peut s'expliquer ici, soit par une mutation délétère du gène (métaboliseurs lents), soit par des mutations qui modifient l'affinité ou la spécificité de l'enzyme, soit enfin par un effet de dosage génique permettant de comprendre pourquoi un faible pourcentage de la population métabolise beaucoup plus rapidement les xénobiotiques, substrats de cette enzyme.

[1. Nelson D, et al. DNA Cell Biol 1993; $12: 1-51$.

[2. Johansson J, et al. Proc Natl Acad Sci USA 1993; 90 : 11825-9.] 